\title{
Sub-1-K Operation of SiGe Transistors and Circuits
}

\author{
Laleh Najafizadeh, Student Member, IEEE, Joseph S. Adams, Stanley D. Phillips, Student Member, IEEE, \\ Kurt A. Moen, Student Member, IEEE, John D. Cressler, Fellow, IEEE, Shahid Aslam, \\ Thomas R. Stevenson, and Robert M. Meloy
}

\begin{abstract}
We present the first measurement results for silicon-germanium (SiGe) heterojunction bipolar transistors (HBTs) and SiGe BiCMOS circuits operating in the sub-1-K regime. Robust transistor operation of a first-generation $0.5 \times$ $2.5 \times 4-\mu \mathrm{m}^{2} \mathrm{SiGe}$ transistor is demonstrated at package temperatures as low as $300 \mathrm{mK}$. In addition, a SiGe BiCMOS bandgap voltage reference is verified to be fully functional at operating temperatures below $700 \mathrm{mK}$. The SiGe voltage reference exhibits a temperature coefficient of $160 \mathrm{ppm} /{ }^{\circ} \mathrm{C}$ over the temperature range of $700 \mathrm{mK}-300 \mathrm{~K}$.
\end{abstract}

Index Terms-Analog integrated circuits, BiCMOS, cryogenic temperatures, heterojunction bipolar transistor (HBT), silicon germanium (SiGe), SiGe HBTs, voltage reference.

\section{Motivation}

$\mathbf{S}$ UBMILLIMETER-WAVELENGTH astronomical instrumentation generally requires readout circuits and detectors that can reliably operate in the deep cryogenic temperature regime (typically sub-1 K) so that thermal noise is strongly suppressed. Analog circuits such as voltage and current references, as well as amplifiers, are essential components of such detectors and readout circuits [1]-[4]. Due to severe limitations in functionality of conventional semiconductor devices at such extremely low temperatures, these requisite electronic circuits are required to be operated at temperatures that are considerably higher than the detector's sub-1-K operating temperature. Therefore, such detector systems, which are often flown into space, necessarily consist of several cryogenic coolers so that multiple temperatures can be provided (one for the electronic circuits and one for other detector components) [2], [4]. If the electronic circuits could be designed to operate at the detector's sub-1-K operating temperature, the need for large numbers of signal wires providing connections between intermediate cryogenic coolers would be eliminated, resulting in dramatic

Manuscript received January 15, 2009; revised February 21, 2009. Current version published April 28, 2009. This work was supported by the National Aeronautics and Space Administration. The review of this letter was arranged by Editor M. Ostling.

L. Najafizadeh, S. D. Phillips, K. A. Moen, and J. D. Cressler are with the School of Electrical and Computer Engineering, Georgia Institute of Technology, Atlanta, GA 30332-0250 USA (e-mail: laleh@ece.gatech.edu; stan.phillips@gatech.edu; kmoen@ece.gatech.edu; cressler@ece.gatech.edu).

J. S. Adams is with The University of Maryland, Baltimore, MD 21250 USA (e-mail: Joseph.S.Adams@ nasa.gov).

S. Aslam is with MEI Technologies, Inc., Lanham, MD 20706 USA, and also with the NASA Goddard Space Flight Center, Greenbelt, MD 20771 USA (e-mail: Shahid.Aslam-1@ nasa.gov).

T. R. Stevenson is with the NASA Goddard Space Flight Center, Greenbelt, MD 20771 USA (e-mail: Thomas.R.Stevenson@nasa.gov).

R. M. Meloy is with MEI Technologies, Inc., Lanham, MD 20706 USA (e-mail: bmeloy@hst.nasa.gov).

Color versions of one or more of the figures in this letter are available online at http://ieeexplore.ieee.org.

Digital Object Identifier 10.1109/LED.2009.2016767 reductions in system weight and volume, and the cost of the overall mission.

Silicon-germanium (SiGe) BiCMOS technology has emerged as a compelling technology platform for implementing electronic circuits intended for extreme environment applications (e.g., when exposed to low temperatures, high temperatures, and/or ionizing radiation) [5]. Due to the beneficial effects of bandgap engineering, the electrical properties of $\mathrm{SiGe}$ transistors such as the current gain, cutoff frequency, and broadband noise all improve with cooling. Below about $100 \mathrm{~K}$, where Si bipolar junction transistors suffer strongly from base freeze-out [6], carrier freeze-out is minimized in SiGe HBTs since the base, emitter, and significant portions of the collector are doped well above $3 \times 10^{18} \mathrm{~cm}^{-3}$, a minimum required doping level in $\mathrm{Si}$ for the occurrence of the semiconductor-metal (Mott) transition [5]-[7]. To date, a substantial amount of the available information on the cryogenic operation of SiGe HBTs and circuits has been limited to a lower temperature bound of about $4.2 \mathrm{~K}$ (liquidhelium temperature) [8]-[14], and no data are available on the capability of SiGe HBTs and circuits for operation below $4.2 \mathrm{~K}$. We point out that many transistor-relevant parameters (e.g., carrier density) are thermally activated [proportional to $\exp (E / k T)]$, and thus, the impact of temperature on the carrier distributions, which determine both current flow and transistor action, between, for example, $4.2 \mathrm{~K}$ and $300 \mathrm{mK}$, is enormous (e.g., if $E=1 \mathrm{eV}$, a change by a factor of $e^{17943}$ theoretically exists in intrinsic carrier concentration between $4.2 \mathrm{~K}$ and $300 \mathrm{mK}$ ). As a result, it is widely believed that $\mathrm{Si}$ bipolar transistors will not function at sub-1-K temperatures. In this letter, we present the first dc measurement results for SiGe HBTs operating in environments as low as $300 \mathrm{mK}$. In addition, the operation of a SiGe bandgap voltage reference (BGR) is fully verified for operation in the sub-1-K regime.

\section{Process Technology and Circuit Description}

The transistors used for this letter are from a first-generation SiGe (IBM's SiGe 5AM) BiCMOS technology, which features SiGe HBTs with an emitter width of $0.5 \mu \mathrm{m}, B V_{\mathrm{CEO}}$ of $3.3 \mathrm{~V}$, and a unity-gain cutoff frequency and a maximum oscillation frequency of 45 and $60 \mathrm{GHz}$ at $300 \mathrm{~K}$, respectively. This four-level metal platform also offers CMOS transistors with a nominal $L_{\text {eff }}$ of $0.35 \mu \mathrm{m}$, as well as polysilicon and diffused resistors, and various capacitors. To fully evaluate the capability of SiGe technology for operation in the sub-1-K regime, an exponentially compensated BGR [15] was implemented. The schematic of the circuit is shown in the inset of Fig. 4, and the dimensions of the transistors and resistors are summarized in Table I. The start-up circuit consists of transistors $\mathrm{M}_{1}, \mathrm{M}_{2}$, and 
TABLE I

DIMENSIONS OF SiGe HBTs AND CMOS TRANSISTORS IN THE BGR CIRCUIT

\begin{tabular}{|l|l|}
\hline $\mathrm{M}_{1}: \quad(\mathrm{W} / \mathrm{L})=300 \mu \mathrm{m} / 1 \mu \mathrm{m}$ & $\mathrm{M}_{10}, \mathrm{M}_{11}:(\mathrm{W} / \mathrm{L})=40 \mu \mathrm{m} / 1 \mu \mathrm{m}$ \\
\hline $\mathrm{M}_{2}, \mathrm{M}_{4}-\mathrm{M}_{7}:(\mathrm{W} / \mathrm{L})=20 \mu \mathrm{m} / 1 \mu \mathrm{m}$ & $\mathrm{Q}_{1}, \mathrm{Q}_{3}, \mathrm{Q}_{4}, \mathrm{Q}_{5}: \mathrm{A}_{\mathrm{E}}=4 \times 0.5 \times 2.5 \mu \mathrm{m}^{2}$ \\
\hline $\mathrm{M}_{3}:(\mathrm{W} / \mathrm{L})=2 \mu \mathrm{m} / 90 \mu \mathrm{m}$ & $\mathrm{Q}_{2}: \mathrm{A}_{\mathrm{E}}=32 \times 0.5 \times 2.5 \mu \mathrm{m}^{2}$ \\
\hline $\mathrm{M}_{8}, \mathrm{M}_{9}:(\mathrm{W} / \mathrm{L})=10 \mu \mathrm{m} / 1 \mu \mathrm{m}$ & $\mathrm{R}_{1}=2 \mathrm{k} \Omega, \mathrm{R}_{2}=15.4 \mathrm{k} \Omega$ \\
\hline
\end{tabular}

$\mathrm{M}_{3}$. Transistors $\mathrm{Q}_{1}$ and $\mathrm{Q}_{2}$, along with resistor $\mathrm{R}_{1}$, generate a proportional-to-absolute-temperature (PTAT) current for the following stages. This PTAT current is mirrored and amplified through the pMOS current mirrors and transistor $\mathrm{Q}_{5}$ into the last two stages, generating a PTAT voltage across resistor $\mathrm{R}_{2}$ that compensates the negative temperature coefficients of the base-emitter voltage of transistor $\mathrm{Q}_{3}$, creating a temperaturestable output voltage.

\section{EXPERIMENTAL SETUP}

Device structures and the $\mathrm{SiGe}$ voltage reference were mounted into a 48-pin ceramic flat package and wire bonded. The package was epoxied into a copper sample holder that was then mounted on the copper cold stage of a pumped ${ }^{3} \mathrm{He}$ refrigerator capable of reaching a base temperature of less than $300 \mathrm{mK}$ under a $40-\mu \mathrm{W}$ heat load. A calibrated ruthenium oxide temperature sensor was located nearby to obtain the temperature of the cold stage. In addition, a Cernox temperature sensor, CX-1010-BR [16], was mounted adjacent to the die in the package to closely monitor the die's temperature during the experiment. This temperature sensor is a sputter-deposited thin-film resistor with a negative temperature coefficient. The measured resistance of the Cernox resistor as a function of the ${ }^{3} \mathrm{He}$-pot temperature, at $252 \mathrm{mK}$, and for a temperature range of $400-800 \mathrm{mK}$, is shown in Fig. 1. A photomicrograph showing the location of the sensor and die in the package is shown in the inset of Fig. 1. It can be seen that a sensor resistance value of $600 \Omega$ or larger corresponds to die temperatures lower than $800 \mathrm{mK}$. Transistor dc characterization was performed using an Agilent 4155 Semiconductor Parameter Analyzer. The output voltage of the BGR circuit was recorded as a function of temperature, during both cooldown and warm-up, using precision Agilent meters.

\section{EXPERIMENTAL RESULTS}

The refrigerator reached a base temperature of $252 \mathrm{mK}$ with no extra heat load injected into the system. The system was maintained in this state for $50 \mathrm{~min}$ prior to performing $\mathrm{dc}$ characterization to ensure equilibration. In order to verify the functionality of SiGe HBTs at this base temperature, forced- $I_{B}$ output characteristics were measured at nanoampere-level currents to establish transistor action. During these measurements, the total power injected into the system was kept below $40 \mu \mathrm{W}$. Shown in Fig. 2 are the output characteristics of the SiGe HBT taken at $4.0 \mathrm{~K}, 1.4 \mathrm{~K}$, and $252 \mathrm{mK}$ for base currents of 40-100 nA. The package temperature (as read out by the Cernox sensor) remained below $300 \mathrm{mK}$ (Fig. 1). As can be seen, clear transistor functionality is observed, and at the base current of $100 \mathrm{nA}$, a maximum current gain of 9.2 is achieved at package temperatures lower than $300 \mathrm{mK}$. At a $V_{\mathrm{CE}}$ of $1.0 \mathrm{~V}$,

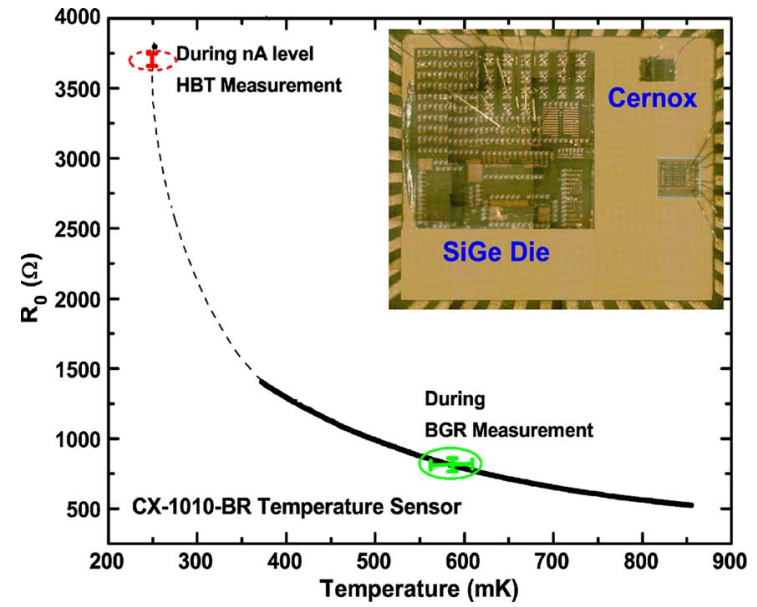

Fig. 1. Measured characteristics of the on-package Cernox resistor as a function of the measured ${ }^{3} \mathrm{He}$-pot temperature.

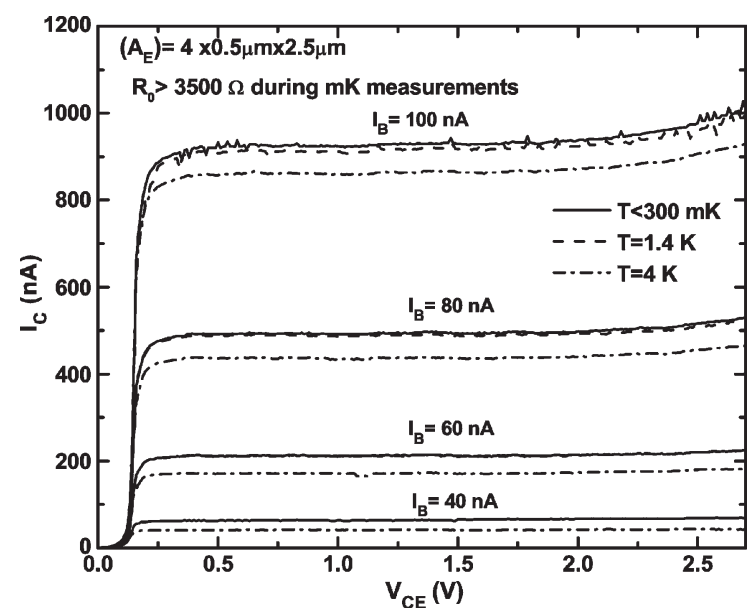

Fig. 2. Measured forced- $I_{B}$ output characteristics of a $4 \times 0.5 \times 2.5 \mu \mathrm{m}^{2}$ $\mathrm{SiGe} \mathrm{HBT}$ at $4 \mathrm{~K}, 1.4 \mathrm{~K}$, and below $300 \mathrm{mK}$.

and $I_{B}$ of $100 \mathrm{nA}$, the power dissipated by the SiGe HBT is estimated to be around $1 \mu \mathrm{W}$ at this temperature. Forced- $I_{B}$ output characteristics at higher base current levels, as well as the full Gummel characteristics, were also measured, and the transistor showed reasonably ideal behavior. However, due to the injection of relatively large amounts of heat during these measurements, a temperature rise was observed in the package. Fig. 3 shows the measured output voltage of the SiGe BGR circuit operating over a 1200 -s window at a sub-1-K operating temperature. The average output voltage obtained during this period was $1.1562 \mathrm{~V}$, and the voltage deviation from the average value was less than $800 \mu \mathrm{V}$. With a $3.3-\mathrm{V}$ power supply, the circuit consumes an average current of $39.5 \mu \mathrm{A}$ and dissipates about $130 \mu \mathrm{W}$. During the measurements, the package temperature stayed below $700 \mathrm{mK}$ (Fig. 1). The observed fluctuations in the output voltage can be further decreased by adding an onchip capacitor at the output node. The measured output voltage as a function of package temperature is shown in Fig. 4. As explained in the previous section, the output voltage of the BGR circuit can be estimated to be

$$
V_{\mathrm{out}} \approx V_{\mathrm{BE}, Q 3}+\frac{\Delta V_{\mathrm{BE}}}{R_{1}}\left(k_{1} R_{2}+\frac{k_{2} R_{2}}{\beta_{Q 3}}\right)
$$




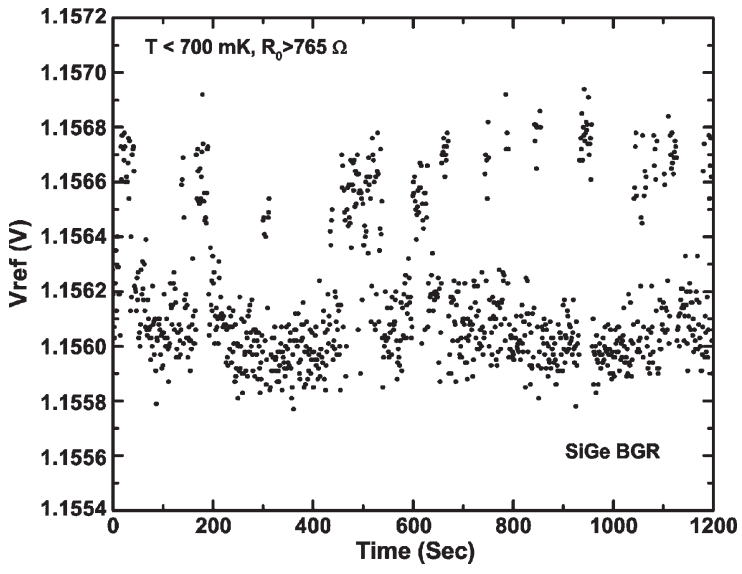

Fig. 3. Measured output voltage of a SiGe BGR over 1200-s window when operating at temperatures below $700 \mathrm{mK}$.

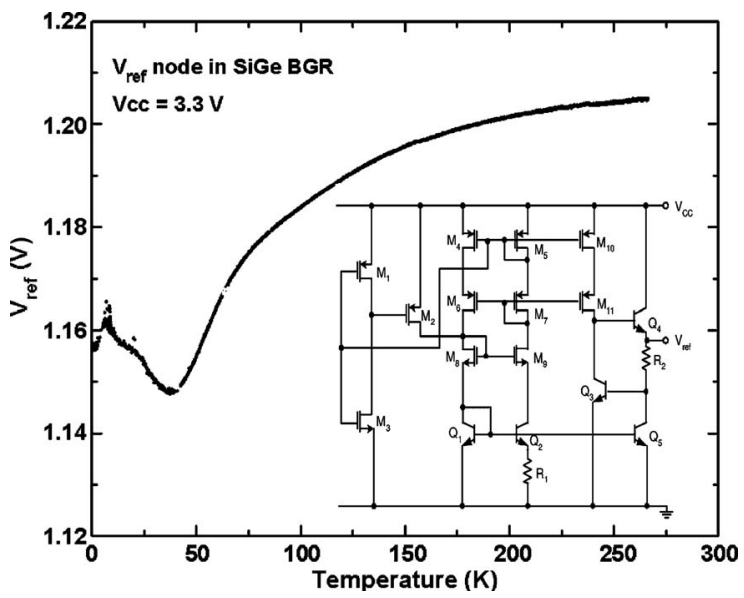

Fig. 4. Measured output voltage (1526900 points) of the SiGe BGR as a function of temperature.

where $V_{\mathrm{BE}, Q 3}$ is the base-emitter voltage of transistor $\mathrm{Q}_{3}$, which is a decreasing function of temperature; $\Delta V_{\mathrm{BE}}$ is the PTAT voltage, which is generated by the voltage difference between the base-emitter voltages of transistors $\mathrm{Q}_{1}$ and $\mathrm{Q}_{2}$; $k_{1}$ and $k_{2}$ are the amplification factors of the PTAT current in the last two stages; and $\beta_{Q 3}$ is the current gain of $\mathrm{Q}_{3}$. The circuit was originally designed and optimized for the mil-spec temperature range (from $-55^{\circ} \mathrm{C}$ to $+125^{\circ} \mathrm{C}$ ). As the temperature drops below $220 \mathrm{~K}$, the rate of increase in the base-emitter voltage is dominated by the rate of decrease in the PTAT voltage, and as a result, the output voltage decreases as the temperature is reduced. Below $36 \mathrm{~K}$, however, the output voltage begins to rise. This is due to the fact that the nonlinearity in the temperature variation of the base-emitter voltage becomes more severe at these extremely low temperatures [17] and that its first-order temperature coefficient is therefore decreased. As a result, the $\Delta V_{\mathrm{BE}}$ voltage [second term in (1)] will no longer vary linearly with temperature, and instead, it becomes a weak function of temperature. Therefore, the output voltage starts to increase, as the negative temperature coefficients of $V_{\mathrm{BE}, Q 3}$ become the dominant factor. Although we expect this trend to continue down to millikelvin temperatures, a slight decrease in the output voltage is observed when the temperature is further decreased below about $4 \mathrm{~K}$. More investigation is required to understand this behavior. Fig. 4 verifies that the BGR circuit is fully functional across the temperature range of $700 \mathrm{mK}-300 \mathrm{~K}$, with a temperature coefficient of $160 \mathrm{ppm} /{ }^{\circ} \mathrm{C}$.

\section{SUMMARY}

We have demonstrated the first dc measurements of SiGe HBTs operating in environments below $1 \mathrm{~K}$. The SiGe HBTs remain functional, with usable current gain. A SiGe BGR circuit was also fully characterized and verified to operate reliably at sub-1-K temperatures.

\section{ACKNOWLEDGMENT}

The authors would like to thank S. Babu, the NASA JWST team, and the NASA SiGe ETDP team for their contributions.

\section{REFERENCES}

[1] T. Cunningham and E. Fossum, "GaAs JFETs intended for deep cryogenic VLWIR readout electronics," J. Phys., IV, vol. 4, pp. 147-152, Jun. 1994.

[2] M. Fujiwara and M. Sasaki, "Performance of GaAs JFETs at a cryogenic temperature for application to readout circuit of high-impedance detectors," IEEE Trans. Electron Devices, vol. 51, no. 12, pp. 2042-2047, Dec. 2004.

[3] P. Merken, T. Souverijns, J. Putzeys, Y. Creten, and V. Van Hoof, "Low noise, low power readout electronics circuit development in standard CMOS technology for 4 K applications," Proc. SPIE, vol. 6275, no. 6284, pp. 627 516-1-627 516-8, Jul. 2006.

[4] H. Nagata, J. Kobayashi, H. Matsuo, and M. Fujiwara, "Progress on GaAs cryogenic readout circuits for SISCAM," Proc. SPIE, vol. 6275, no. 6284, pp. 627 527-1-627 527-10, Jul. 2006.

[5] J. D. Cressler, "On the potential of SiGe HBTs for extreme environment electronics," Proc. IEEE, vol. 93, no. 9, pp. 1559-1582, Sep. 2005.

[6] J. D. Cressler and G. Niu, Silicon-Germanium Heterojunction Bipolar Transistors. Norwood, MA: Artech House, 2003.

[7] R. S. Popovic, Hall Effect Devices, 2nd ed. Boca Raton, FL: CRC Press, 2004.

[8] A. J. Joseph, J. D. Cressler, and D. M. Richely, "Operation of SiGe heterojunction bipolar transistors in liquid-helium temperature regime," IEEE Electron Device Lett., vol. 16, no. 6, pp. 268-270, Jun. 1995.

[9] R. Krithivasan, Y. Lu, J. D. Cressler, J.-S. Rieh, M. H. Khater, D. Ahlgren, and G. Freeman, "Half-terahertz operation of SiGe HBTs," IEEE Electron Device Lett., vol. 27, no. 7, pp. 567-569, Jul. 2006.

[10] J. Yuan, R. Krithivasan, J. D. Cressler, M. H. Khater, D. Ahlgren, and A. J. Joseph, "On the frequency limits of SiGe HBTs for terahertz applications," in Proc. IEEE Bipolar/BiCMOS Circuits Technol. Meeting, Oct. 2007, pp. 22-25.

[11] P. Chevalier, N. Zerounian, B. Barbalat, F. Aniel, and A. Chantre, "On the use of cryogenic measurements to investigate the potential of $\mathrm{Si} / \mathrm{SiGe}: \mathrm{C}$ HBTs for terahertz operation," in Proc. IEEE Bipolar/BiCMOS Circuits Technol. Meeting, Oct. 2007, pp. 26-29.

[12] N. Zerounian, F. Aniel, B. Barbalat, P. Chevalier, and A. Chantre, "500 GHz cutoff frequency SiGe HBTs," Electron. Lett., vol. 43, no. 14, pp. 774-775, Jul. 2007.

[13] L. Najafizadeh, M. Bellini, A. P. G. Prakash, G. A. Espinel, J. D. Cressler, P. W. Marshall, and C. J. Marshall, "Proton tolerance of SiGe precision voltage references for extreme temperature range electronics," IEEE Trans. Nucl. Sci., vol. 53, no. 6, pp. 3210-3216, Dec. 2006.

[14] R. Krithivasan, Y. Lu, L. Najafizadeh, C. Zhu, J. D. Cressler, S. Chen, C. Ulaganathan, and B. J. Blalock, "A high-slew rate SiGe BiCMOS operational amplifier for operation down to deep cryogenic temperatures," in Proc. IEEE Bipolar/BiCMOS Circuits Technol. Meeting, Oct. 2006, pp. $72-75$.

[15] I. Lee, G. Kim, and W. Kim, "Exponential curvature-compensated BiCMOS bandgap references," IEEE J. Solid-State Circuits, vol. 29, no. 11, pp. 1396-1403, Nov. 1994.

[16] [Online]. Available: http://www.lakeshore.com/temp/sen/crtd.html

[17] Y. Tsividis, "Accurate analysis of temperature effects in $I_{C}-V_{\mathrm{BE}}$ characteristics with application to bandgap reference sources," IEEE J. SolidState Circuits, vol. SSC-15, no. 6, pp. 1076-1084, Dec. 1980. 